\title{
Recuperar bosques no es solo plantar árboles: lecciones aprendidas luego de 7 años restaurando bosques de Pilgerodendron Uviferum (D. Don) Florin en Chiloé
}

\author{
Forest restoration is more tan tree planting: lessons learned after \\ restoring Pilgerodendron uviferum forests during 7 years in Chiloé
}

Jan R. Bannister"

\section{Resumen}

Hace 7 años se inició un proyecto de investigación a largo plazo cuyo principal objetivo fue el estudiar bosques turbosos inalterados y quemados de Pilgerodendron uviferum en el sur de Chiloé, de tal forma de desarrollar la base ecológicacientífica necesaria para futuras estrategias de conservación y restauración. Se enfocó el trabajo en tres importantes aspectos relacionados a la restauración: a) entender los procesos ecológicos que ocurren en bosques turbosos inalterados, b) analizar el grado de recuperación natural de los bosques quemados, y c) explorar algunas opciones para su restauración. Según nuestros resultados, pese a ser $P$. uviferum una especie tolerante al estrés, su recuperación luego de incendios es extremadamente lenta. Setenta años después de incendios catastróficos, a escala de paisaje existe una muy baja frecuencia de árboles semilleros y su potencial de diseminación de semillas es extremadamente limitado. Los resultados de este estudio revelan que incendios severos pueden prácticamente eliminar la especie de extensas zonas del paisaje. Además, destaca la importancia de la persistencia de legados biológicos, como son los árboles semilleros, para la recuperación de sitios alterados. Por este motivo, plantaciones con la especie como complemento a los árboles semilleros, las cuales toleran condiciones de humedad extrema, pero sufren por estrés cuando crecen en zonas descubiertas, podrían ayudar a asistir la recuperación natural de esta especie y sumar diversidad genética. En este contexto, se sugiere una estrategia mixta pasiva-activa de restauración, basada en regeneración natural proveniente de árboles semilleros y la plantación complementaria en clusters. Finalmente, el enfoque multi-escala usado, en el cual se estudian los procesos ecológicos y fisiológicos esenciales que ocurren en sitios alterados e inalterados de forma previa a la planificación de las actividades de restauración, puede ser adoptado para otros ecosistemas con baja resiliencia y alta degradación, donde la restauración es extremadamente costosa $y$ sus resultados inciertos.

\section{Palabras clave:}

Bosques turbosos, árboles semilleros, legados biológicos, estrategia mixta de restauración, patrones espaciales.

\begin{abstract}
Seven years ago we commenced a long-term research project on Chiloé Island, North Patagonia, dealing with the study of disturbed and undisturbed Pilgerodendron uviferum forests, in order to develop the ecological basis for future conservation and restoration strategies of the species. Using a multi-scaled approach, we focussed research on three aspects of restoration a) understanding the ecological processes occurring in undisturbed forests of the species; b) analysing the natural rate of recovery of disturbed forests of the species, and c) exploring some options for the restoration of
\end{abstract}

Instituto Forestal, Chile, tel.: +56-65-2633641,

jbannister@infor.cl $\$ 
these forests. Our results show that $P$. uviferum is a stress-tolerant conifer that can tolerate extremely wet conditions, yet suffer from stress when grown in the open, and therefore the recovery of the species after fires is extremely slow. At the landscape level, seventy years after stand replacing fires there are vast areas without seed trees of the species, and their dissemination potential is extremely limited. Therefore, restoration planting to complement existing seed trees may assist natural recovery of $P$. uviferum in disturbed bog forests and add genetic diversity. In this context, we suggest a mixed passive-active restoration strategy, relying on naturally regenerated parent trees complemented through cluster planting. Furthermore, the multi-scale approach used in our research, that studied the underlying ecological and physiological processes occurring in disturbed and undisturbed sites prior to planning a restoration program, could be adopted for other ecosystems with low resilience and high degradation, where restoration is likely to be extremely expensive and the outcome uncertain.

\section{Key words:}

Bog forests, seed tres, biological legacies, mixed restoration strategies, spatial patterns.

\section{INTRODUCCIÓN}

Los humedales boreales y árticos cubren una superficie de casi 3,5 millones de $\mathrm{km}^{2}$ (Shaw et al. 2003). Debido al desequilibrio entre las tasas de fijación de carbono y los procesos de descomposición en estos ecosistemas, estos acumulan grandes cantidades de turba. Se estima que un tercio del carbono mundial acumulado está guardado en turberas boreales (Gorham, 1991). Mientras la mayoría de los humedales y turberas a nivel mundial están concentrados en la zona boreal, millones de hectáreas de estos ecosistemas se pueden encontrar también en zonas subantárticas de Australia, Nueva Zelanda y el cono sur Sudamericano (Chile y Argentina) (Shaw et al. 2003).

Pese a la gran superficie de turberas existente en el cono sur de Sudamérica (específicamente la Patagonia), existen escasos estudios sobre la estructura, dinámica y estado de conservación de los bosques turbosos australes allí ubicados, los cuales son dominados generalmente por la conífera Pilgerodendron uviferum (D. Don) Florin (Ciprés de las Guaitecas). Es más, estos bosques turbosos son uno de los bosques templados costeros menos estudiados del sur de Sudamérica (Holz \& Veblen, 2009), particularmente en su estado adulto inalterado (Bannister et al. 2008). La presión antrópica sobre estos bosques para la extracción de madera ha causado la destrucción extensiva de estos "cipresales" a través de la quema para facilitar el acceso a los árboles (Lara et al. 2014; Bannister et al. 2008). La importancia de la madera de $P$. uviferum en el contexto histórico es demostrada por el establecimiento durante el siglo XIX de la industria forestal en la Isla de Chiloé y el Archipiélago de Las Guaitecas (Patagonia Norte). Producto de la industria forestal de esos años se llegaron a producir sobre 300.000 durmientes anuales, los cuales fueron vendidos para abastecer el sistema ferroviario del norte de Chile y Perú (Otero, 2006). La utilización histórica de esta especie acompañada de talas ilegales en reservas naturales son problemas constantes para la conservación de la especie en la zona austral de Chile (Bannister, 2004; Szeicz et al. 2000). Pese a esto, actualmente los bosques dominados por $P$. uviferum, cubren un área de 930.074 ha desde los $40^{\circ} \mathrm{S}$ y los $55^{\circ} \mathrm{S}$, representando el $6.8 \%$ de los bosques nativos de Chile (CONAF, 2011). Esta enorme superficie aumenta significativamente si se consideran todas las comunidades que presentan esta especie, y no solamente el tipo forestal (Donoso, 1981), ya que una gran extensión de éstos bosques no son oficialmente considerados dentro del tipo forestal ( $<10$ árboles/ha y $<2 \mathrm{~m}$ altura). Sin embargo, los bosques turbosos dominados por $P$. uviferum representan un típico caso de ecosistema con baja resiliencia a incendios, en los cuales se queman gran parte de los árboles y semillas (Bannister, 2012), por lo cual luego de alteraciones extensivas estos bosques quemados no pueden retornar a bosques dominados por P. uviferum (Bannister et al. 2008; Cruz \& Lara, 1981).

Esto contrasta con el gran valor ecológico, económico y social de estos bosques al ser importantes como sumideros de carbono (Gorham, 1991), para la regulación del ciclo hidrológico (Bullock \& Acreman, 2003) y por estar ubicados 
en un hotspot mundial de biodiversidad no vascular (Rozzi et al. 2008). Su especie dominante, P. uviferum es una especie que fue clasificada por IUCN como vulnerable (Walter \& Gillet, 1998) e incluida en el Apéndice I de CITES (Hechenleitner et al. 2005; Soto et al. 2010). Además, los árboles de $P$. uviferum son reconocidos por ser archivos climáticos naturales (Holz \& Veblen, 2009) y actualmente existe una alta demanda por su madera, la cual es muy valorada para todo tipo de construcciones, embarcaciones y postes de cercos (Bannister et al. 2008).

En este contexto, hace 7 años se inició un programa de investigación a largo plazo entre el Instituto de Silvicultura de la Universidad de Freiburg, Alemania y el Parque Tantauco en Chiloé, cuyo principal objetivo fue el estudiar bosques turbosos inalterados y quemados de P. uviferum en el sur de Chiloé, de tal forma de desarrollar la base ecológica-científica necesaria para futuras estrategias de conservación y restauración. Durante todos estos años se ha enfocado el trabajo en tres importantes aspectos relacionados a la restauración: a) entender los procesos ecológicos que ocurren en bosques turbosos inalterados, b) analizar el grado de recuperación natural de los bosques quemados, y c) explorar algunas opciones para su restauración. De este programa de investigación ha surgido información para diversas publicaciones científicas en los últimos años (Bannister, 2012; Bannister et al. 2013, 2014; Bannister et al. 2012) y capítulos de libros (Lara et al. 2013, 2014). En el presente trabajo se pretende resumir los principales resultados, experiencias, triunfos y fracasos tratando de estudiar y restaurar bosques alterados de $P$. uviferum. A continuación se empieza presentando aspectos generales sobre la ecología y dinámica de $P$. uviferum e información sobre la resiliencia de la especie al fuego, para luego discutir sobre enfoques de restauración para estos bosques y llegar a la definición de una estrategia de restauración para ellos.

\section{ASPECTOS GENERALES SOBRE LA ECOLOGÍA Y DINÁMICA DE LOS BOSQUES DE PILGERODENDRON UVIFERUM}

Pilgerodendron uviferum es una conífera endémica y dioica de la Patagonia. Es la conífera de distribución más austral del planeta (Martinez, 1981) y es considerada una especie longeva y de lento crecimiento que puede vivir sobre 900 años (Bannister et al. 2012). Sus anillos de crecimiento, los cuales son muy bien reconocibles, hacen a $P$. uviferum una especie ideal para estudios dendrocronológicos (Aravena, 2007; Gatica et al. 2014; Holz \& Veblen, 2009; Roig \& Boninsegna, 1991). Además, la madera y corteza de $P$. uviferum contienen metabolitos secundarios los cuales inhiben bacterias y hongos, lo que aumenta significativamente la resistencia a la pudrición (Solís et al. 2004), aumentando su valor para la construcción (Bannister et al. 2008).

En contraste a las otras coníferas de la Patagonia que crecen en suelos terrestres (e.g. Araucaria araucana, Austrocedrus chilensis, Fitzroya cuipressoides), los bosques de P. uviferum son encontrados en paisajes y sustratos moldeados por la glaciación (transición glacial-postglacial ca. 13.000 años AP), en sitios extremadamente húmedos, con precipitaciones muy altas (2500 a $8000 \mathrm{~mm}$ /año) y sobre suelos ácidos y muy pobres en nutrientes, frecuentemente con horizontes Gley (Bannister, 2012; Villagrán, 1988). Por este motivo, esta conífera es asociada frecuentemente con turberas dominadas por Sphagnum (Lara et al. 2013). En este contexto, los bosques dominados por $P$. uviferum se pueden dividir de forma general en dos tipos topográficos: "bosques turbosos" y "bosques de monte" (Fig. 1). Los bosques turbosos están ubicados en sectores planos, anegados, sobre turberas dominadas preferentemente por Sphagnum y donde $P$. uviferum desarrolla pequeños diámetros y alturas de hasta $5 \mathrm{~m}$. Por el contrario, los bosques de monte se encuentran ubicados en lomas, laderas o sitios de mejor drenaje pero igualmente con presencia de una alta cobertura de briófitas, desarrollan grandes áreas basales, alturas de hasta $20 \mathrm{~m}$ y una estructura vertical rica en doseles, acompañada de otras especies como Podocarpus nubigena Lindl., Tepualia stipularis (Hook et Arn.), Nothofagus nitida (Phil) Krasser., Nothofagus betuloides (Mirb) Oerst., Drimys winteri J.R. et G. Forster. y Weinmannia trichosperma Cav. (Bannister et al. 2012).

Luego de los primeros estudios en este tipo de bosques (Bannister, 2004; Cruz \& Lara, 1981), se 

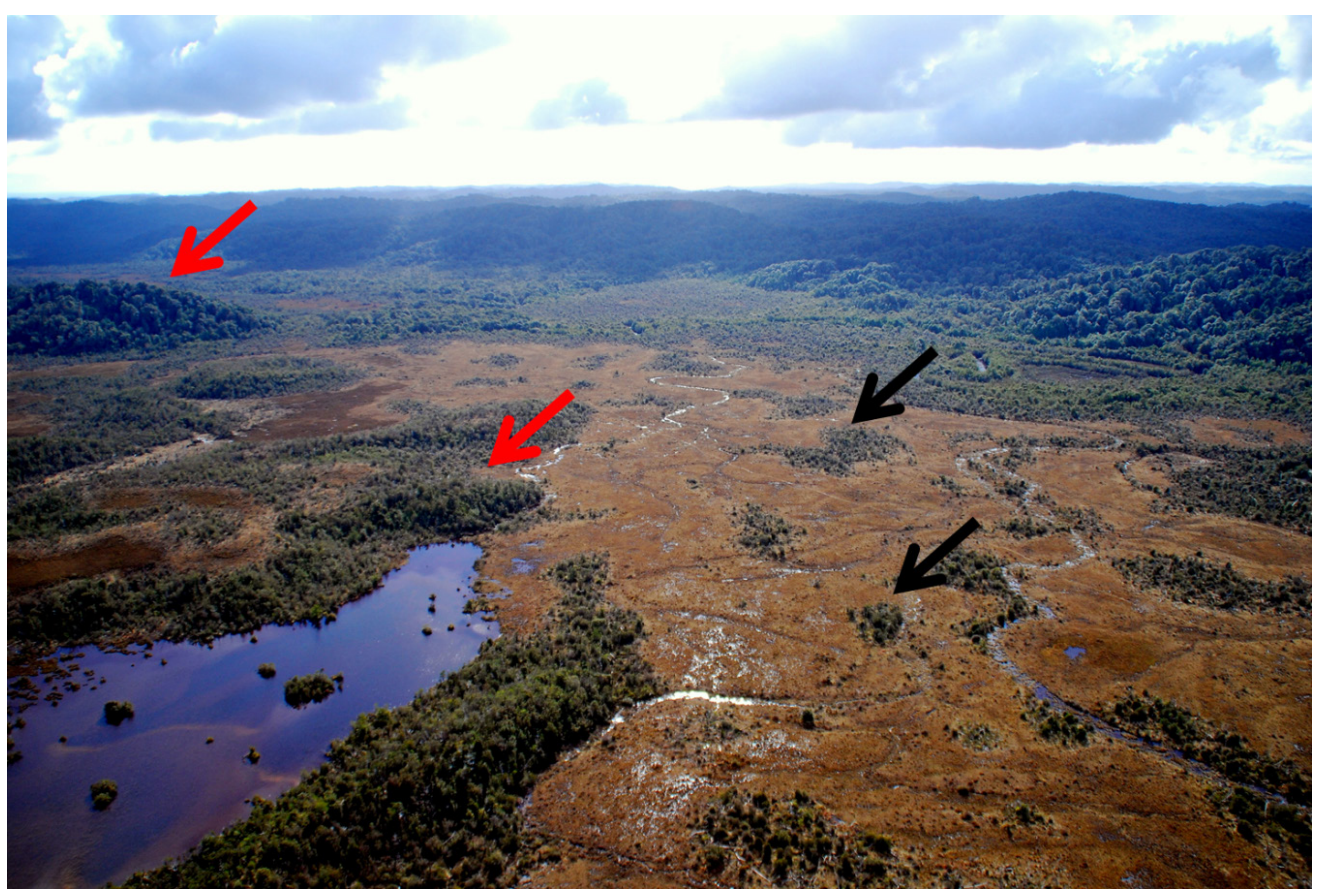

Fig. 1. Tipos de bosques dominados por Pilgerodendron uviferum en el valle del Río Zorra, Parque Tantauco. En general existen bosques de monte (flechas rojas) y bosques turbosos (flechas negras). Fotografía: Alan Bannister.

postuló que su sucesión natural estaba íntimamente relacionada con un mejoramiento de las condiciones de drenaje del suelo, y a medida que avanzaba el tiempo $P$. uviferum iría siendo desplazada por especies acompañantes mejor adaptadas a condiciones favorables de sitio. Sin embargo, se ha demostrado que en bosques turbosos de zonas boreales, en ausencia de alteraciones catastróficas, procesos de paludificación (acumulación de materia orgánica) llevan a estos sitios a perder productividad a medida que pasa el tiempo y no ha mejorarla (Fenton et al. 2005; Lavoie et al. 2005; Simard et al. 2007). En este sentido la acumulación de turba está controlada mayoritariamente por factores autogénicos (sucesión) y la reducción por factores alogénicos como los incendios (Simard et al. 2007), lo cual se contradice con la idea inicial de sucesión natural para los bosques dominados por $P$. uviferum. En este contexto, actualmente se postula que $P$. uviferum es una especie extremamente tolerante al estrés, semitolerante a la sombra, puede crecer a tasas muy bajas, puede regenerarse en ausencia de grandes alteraciones catastróficas, y por este motivo la especie puede persistir a lo largo del tiempo en bosques sucesionalmente avanzados
(Bannister et al. 2012). De esta forma en ausencia de alteraciones catastróficas, la dinámica de $P$. uviferum se desarrollaría de forma diferente en los bosques de monte y en los bosques turbosos (Fig. 2). Según el modelo de dinámica presentado por Bannister (2012), en bosques turbosos, $P$. uviferum coloniza los sectores de mejor drenaje ("hummocks"), se establece y desarrolla. Con el transcurrir de los años, la especie desarrolla una estructura multietánea con abundante regeneración la cual compite permanentemente con briófitas y los efectos de la paludificación. Debido a lo extremo del sitio, su máxima expresión es este tipo de estructura, con árboles de pequeñas dimensiones pero de muy avanzada edad (Cruz \& Lara, 1981). En laderas o lomas extremadamente húmedas pero con mejor drenaje y menor competencia de briofitas, se desarrollan los bosques de monte. Como las condiciones de sitio son mejores que en los bosques turbosos, estos bosques desarrollan mayores áreas basales y existe una mayor cantidad de especies compartiendo el dosel. En estos bosques, la tolerancia al estrés y el lento crecimiento de P. uviferum permite la persistencia de la especie en el lugar, manteniéndose por siglos 
o milenios por medio de dinámica de claros y en base a bajas cantidades de regeneración natural (Bannister et al. 2012).

\section{RESILIENCIA DE PILGERODENDRON UVIFERUM AL FUEGO}

Los disturbios son una parte integral de la dinámica de los ecosistemas forestales. Los humanos alteran los regímenes de perturbación de manera significativa, incrementando su escala y severidad, y reduciendo la resiliencia de los ecosistemas (Walker, 2011). La resiliencia de un ecosistema ha sido definida en términos de la magnitud de disturbios que puede ser absorbida por un ecosistema antes de cambiar de estado, o como el tiempo necesario luego de una perturbación para que un ecosistema retorne a su estado previo. Por este motivo, una posible medida de la resiliencia es qué tanto se ha movido un ecosistema desde su equilibrio y cuánto tiempo necesita para volver (Gunderson, 2000; Holling, 1973). Además, los ecosistemas forestales pueden ser resistentes, lo que representa la capacidad de un ecosistema para absorber disturbios y mantenerse sin grandes cambios (Holling, 1973). La resistencia está relacionada con el concepto de estabilidad, que es la capacidad de un ecosistema de persistir o mantenerse dentro de un equilibrio dinámico en el tiempo mientras resiste cambios (Holling, 1973; Thompson et al. 2009).

De acuerdo al concepto de panarquía de Holling (2001), en un ciclo adaptativo, durante la lenta secuencia desde la fase de explotación a la de conservación, los ecosistemas forestales acumulan biomasa hasta un punto donde la resiliencia es mínima y se transforman en "accidentes esperando suceder". En este contexto, bosques que experimentan raramente disturbios de gran escala son especialmente sensitivos, particularmente si son tipos de disturbios con los cuales los bosques no han evolucionado (Burns, 1993; Parish \& Antos, 2006). Esto es lo que sucede con los bosques dominados por $P$. uviferum, los cuales acumulan grandes cantidades de biomasa con sotobosques dominados por $T$. stipularis y otras

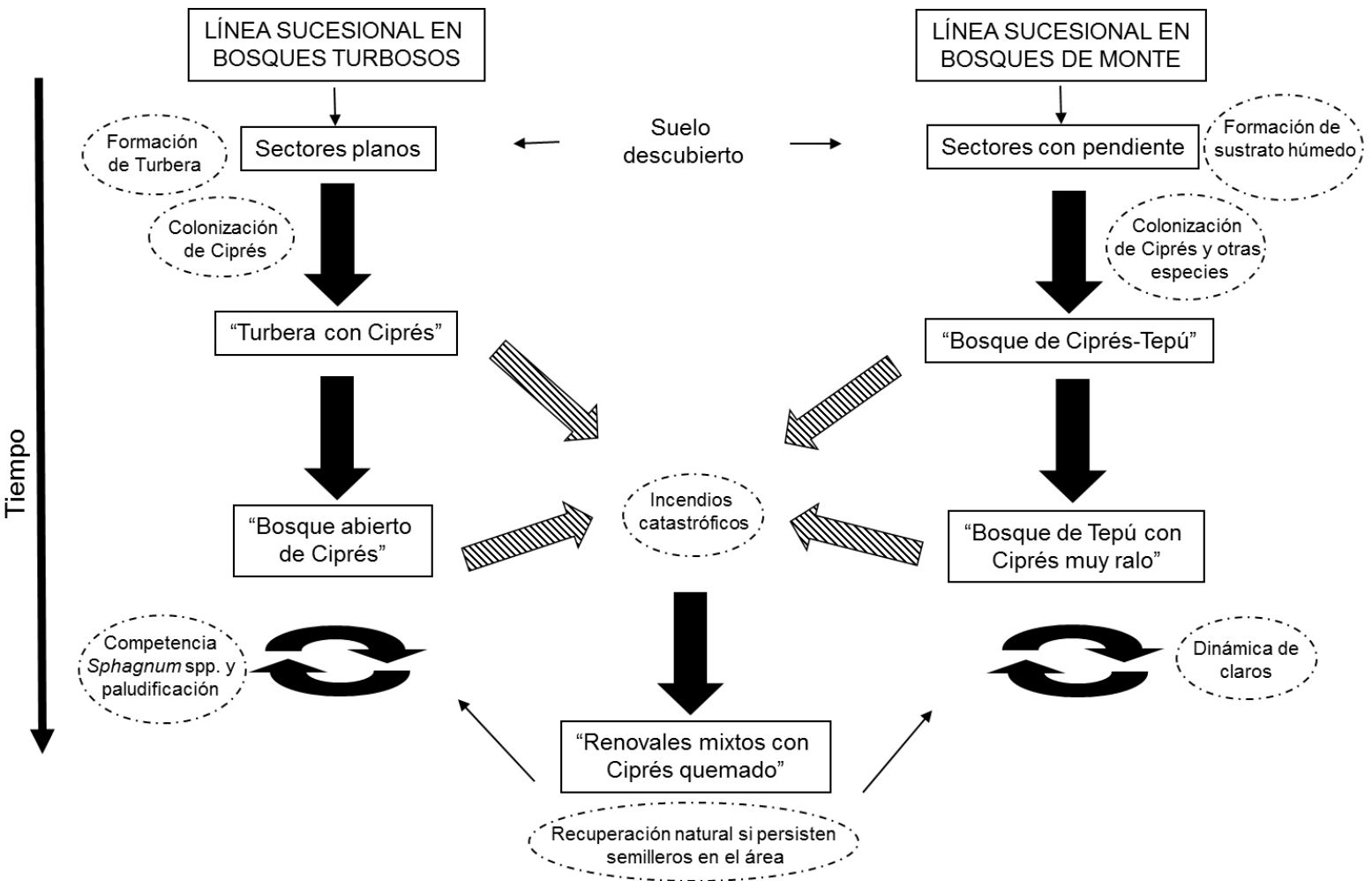

Fig. 2. Modelo de dinámica de bosques de Pilgerodendron uviferum. Extraído y modificado de Bannister (2012) y Lara et al. (2014). 
mirtáceas, los cuales son muy densos, complejos $e$ inmensamente combustibles. Estos sotobosques son el resultado de largos períodos de tiempo sin disturbios que reemplacen el rodal (Bannister, 2012). Por ejemplo, en la Península de Taitao $\left(46^{\circ}\right.$ S) existe registrado una secuencia de polen de aproximadamente 10,000 años desde la última glaciación con presencia relativamente continua de P. uviferum en el rodal (Lumley \& Switsur, 1993). Por este motivo, en el ambiente altamente lluvioso y húmedo de la Patagonia occidental, los incendios están mayoritariamente restringidos a la actividad humana, aunque a veces, árboles individuales $\mathrm{o}$ en pequeños grupos pueden ser afectados por relámpagos (Holz \& Veblen, 2009). Sin embargo, luego de la llegada de colonos europeos y chilenos a la zona insular en el siglo XVIII y XIX, ha habido un incremento significativo de la frecuencia de incendios en la Patagonia norte (Holz, 2010). En este contexto, como P. uviferum no ha co-evolucionado acompañado de incendios catastróficos, la especie no presenta estructuras para soportar el fuego (e.g. corteza gruesa), como si sucede con otras coníferas chilenas como $A$. araucana (Burns, 1993). De esta forma, los incendios históricos han causado cambios drásticos en la estructura y composición de estos bosques (Bannister et al. 2008; Cruz \& Lara, 1981).

Afortunadamente, los disturbios naturales raramente eliminan todos los elementos estructurales del rodal anterior. Una gran variedad de estructuras y organismos sobreviven, incluyendo árboles sexualmente maduros, bancos de semillas o regeneración natural. Estas estructuras y organismos son comúnmente llamados "legados biológicos" (Franklin, 1990; Franklin et al. 2002). La cantidad y tipo de legados biológicos difiere enormemente entre disturbios lo que lleva a diferentes puntos de iniciación para el desarrollo estructural de rodales (Franklin et al. 2002). La importancia de los legados biológicos para el proceso de recuperación de los ecosistemas ha sido muy estudiado en bosques del hemisferio norte (e. g. Franklin, 1990; Franklin \& MacMahon, 2000; Hanssen, 2003; Keeton \& Franklin, 2005; Manning et al. 2006; Herrera \& García, 2009; Kashian et al. 2012), lo que contrasta fuertemente con los pocos estudios realizados en bosques de la Patagonia (Bannister, 2012).
Tabla 1. Principales atributos de cipresales quemados a nivel de paisaje en los alrededores del Lago Chaiguata, Parque Tantauco, Chiloé. Extraído y modificado de Bannister et al. (2014).

Árboles de Pilgerodendron uviferum en el paisaje (100 ha)

Número de árboles / 100 ha

Total

$122(100 \%)$

Por tipo de sitio:

Bosque turboso

$65(53,3 \%)$

Bosque de monte

$57(46,7 \%)$

Género

$\begin{array}{rc}\text { Indefinidos } & 8(6,6 \%) \\ \text { Machos } & 59(48,4 \%) \\ \text { Hembra } & 55(45,1 \%) \\ \text { sexual M:H } & 1.07\end{array}$

Estructura

$\begin{array}{cc}\text { Edad (años) } & 29-81 \\ \operatorname{DAP}(\mathrm{cm}) & 2-20.5 \\ \text { Altura }(\mathrm{m}) & 1-9\end{array}$

Desde hace 7 años, se ha evaluado cómo es la resiliencia de los bosques de $P$. uviferum al fuego, estudiado la tasa de recuperación natural del bosque alterado, y cuál es la importancia de los legados biológicos para esta recuperación. Esta información es de gran importancia para decidir estrategias de restauración para la especie. En este contexto, los resultados acumulados indican que $P$. uviferum pese a ser una especie tolerante al estrés, es muy sensible al fuego y su recuperación luego de incendios es extremadamente lenta. Es así como setenta años después de incendios catastróficos en la zona del Lago Chaiguata, existe una muy baja frecuencia de árboles semilleros a escala de paisaje (0,3 árboles/ha, Tabla 1), y éstos están agregados en escalas de hasta $30 \mathrm{~m}$ (Fig. 3). Los árboles semilleros de $P$. uviferum presentan una semillación muy irregular, con períodos de baja y alta producción de semillas. Por ejemplo el año 2010 (año de alta producción) a una distancia menor a 2 m desde el árbol se pudieron encontrar sobre 200 semillas por $\mathrm{m}^{2}$ (Bannister et al. 2014). Además, el potencial de diseminación de semillas y la distancia efectiva de reclutamiento están limitados a $20 \mathrm{~m}$, y las semillas germinan mejor en sustratos húmedos compuestos por Sphagnum y suelo mineral (Bannister et al. 2014). Adicionalmente, 


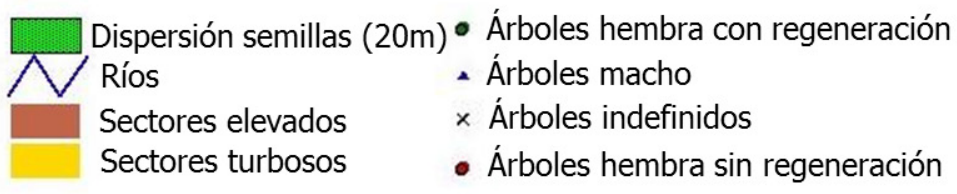

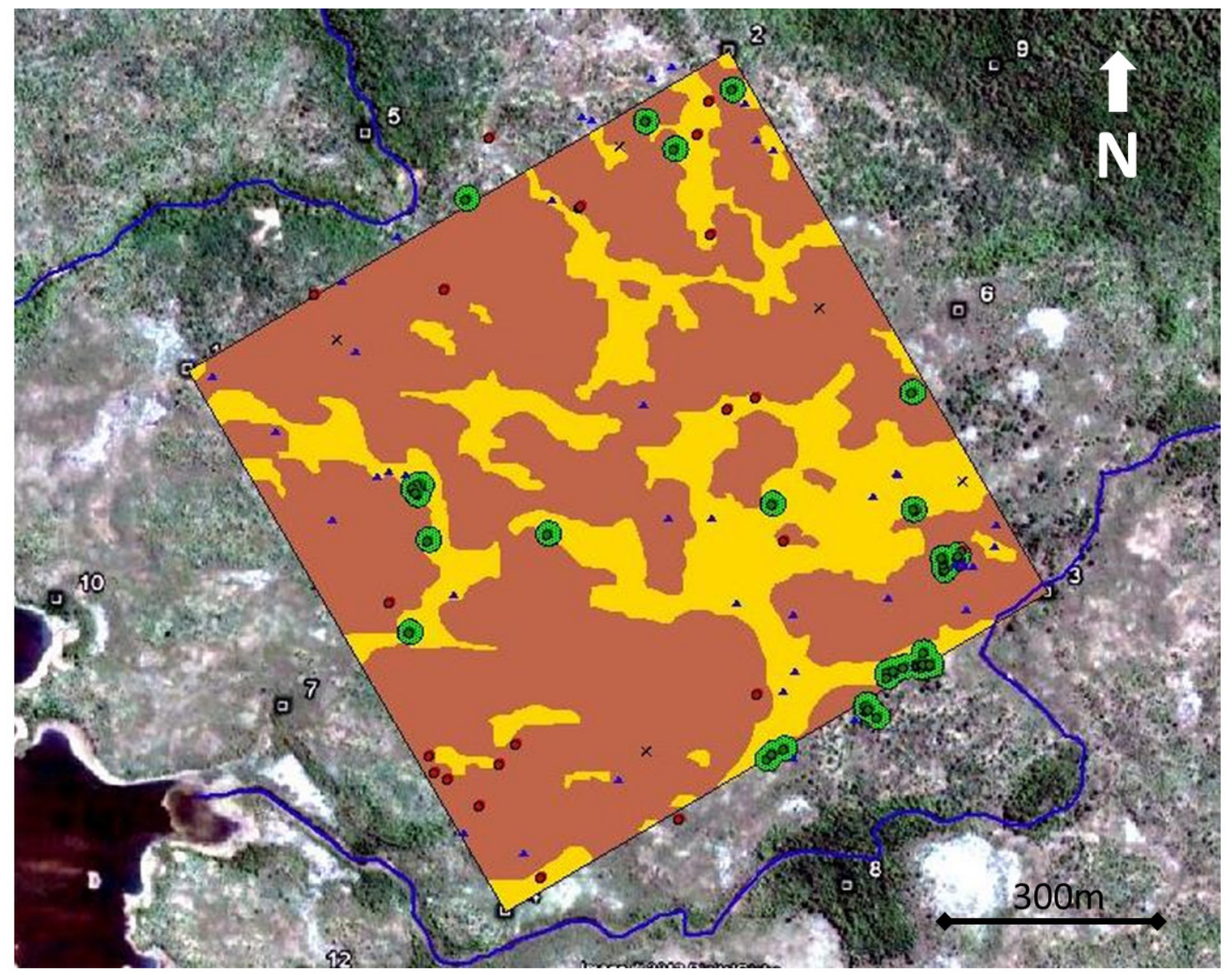

Fig. 3. Diagrama que muestra la distribución de árboles macho y hembra de la conífera Pilgerodendron uviferum a nivel de paisaje (100ha) en un bosque quemado en las orillas del Lago Chaiguata, Parque Tantauco. Círculos verdes representan áreas con regeneración natural de la especie. Extraído y modificado de Bannister et al. (2014).

los vientos tienen un efecto importante en la distribución espacial del reclutamiento de plantas P. uviferum (Fig. 4). Así, los vientos dominantes del área de estudio que vienen desde el Oeste determinan que la regeneración natural se agrupe en dirección este. Estos resultados revelan que incendios severos pueden prácticamente eliminar la especie de extensas zonas del paisaje, donde no sobreviven ni propágalos ni árboles semilleros. Es más, en la zona de estudio, en las cercanías del lago Chaiguata, a escala de paisaje un escaso $3 \%$ del área se está recuperando pasivamente (Bannister et al. 2014). Estos resultados destacan la importancia de la persistencia de legados biológicos, como son los árboles semilleros, para la recuperación de sitios alterados (Fig. 5ac). La regeneración natural post-incendio puede asistir la recuperación de poblaciones alteradas de $P$. uviferum, pero su efecto espacial a nivel de paisaje es extremadamente limitado. Por este motivo, plantaciones con la especie como complemento a los árboles semilleros podrían ayudar a asistir la recuperación natural de $P$. uviferum en bosques turbosos alterados y sumar diversidad genética.

\section{ENFOQUES DE RESTAURACIÓN}

La restauración ecológica es el proceso de 
asistir la recuperación de un ecosistema que ha sido degradado, dañado o destruido (SER, 2004). La ciencia en la cual se basan las acciones de la restauración ecológica es llamada la ecología de la restauración (Van Andel \& Aronson, 2006). El paradigma actual en la ecología de la restauración se enfoca a retornar un ecosistema degradado a algún estado deseado, y dirigir el desarrollo de un ecosistema a lo largo de una trayectoria deseada (Hobbs \& Norton, 1996). Por este motivo, un importante punto de inicio para caracterizar un ecosistema deseado, definir objetivos y desarrollar una estrategia de restauración es tener una referencia (Aronson et al. 1995; Landres et al. 1999; Pickett \& Parker, 1994). Esta "referencia" debiera ser caracterizada en términos de la estructura, dinámica y composición florística que tenían los bosques en condiciones previas a la alteración humana. No obstante, los bosques pueden ser tan variables en el tiempo que no pueden entregar objetivos estáticos de restauración. Una opción para solucionar este problema es una caracterización del rango natural de variabilidad de los bosques y los principales procesos que los influencian (Landres et al. 1999; Kuuluvainen et al. 2002).

Sin embargo, muchas veces no es posible lograr el estado deseado, por lo que se debe buscar y definir estados alternativos. Estos

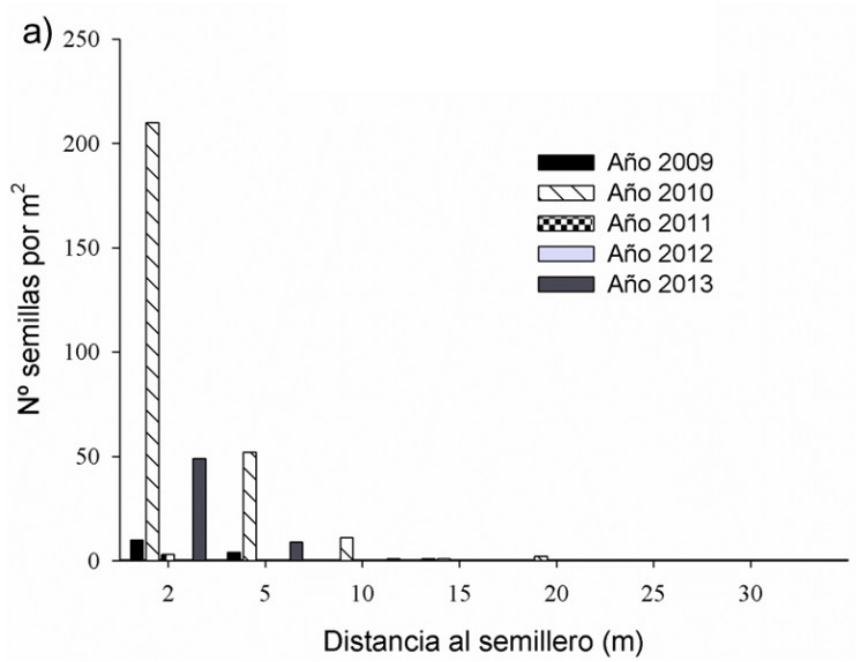

debieran maximizar los niveles de complejidad de tal forma de proveer servicios ecosistemicos similares al ecosistema original. La ventaja de los modelos que incluyen estados alternativos es que reconocen que los ecosistemas pueden moverse abruptamente entre uno o más estados, que la dinámica de un ecosistema degradado es diferente a la del estado original, y que por eso la trayectoria de recuperación va a ser probablemente distinta a la de degradación (Suding et al. 2004). En este contexto, como existen diversos puntos de inicio y objetivos de restauración, en la práctica existen distintos tipos de restauración, entre los cuales se encuentra por ejemplo la reclamación, rehabilitación, reconstrucción y reemplazo (Stanturf et al. 2014).

Además, la restauración de sistemas degradados depende de remover las influencias que definen la degradación, sin embargo esto frecuentemente no es suficiente para promover la restauración (Hobbs \& Norton, 1996). En este contexto, Whisenant (1999) sugiere la existencia de dos tipos de umbrales de restauración los cuales inhiben al sistema a retornar a un estado menos degradado. El primero es un umbral causado por interacciones bióticas y el segundo es controlado por limitaciones abióticas. El tipo de restauración dependerá de cuál de los umbrales se ha cruzado, y la cantidad de esfuerzo necesario para restaurar el sistema variará a lo largo de estos gradientes

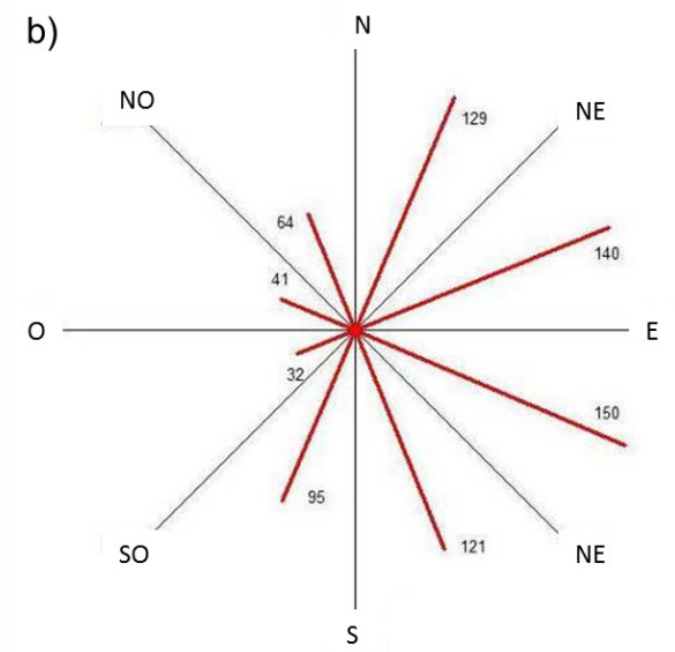

Fig. 4. Dispersión de semillas y distribución de plántulas y brinzales de Pilgerodendron uviferum alrededor de árboles semilleros: (a) Diseminación de semillas por un período de 6 años (año 2014 no hubo semillación); (b) Distribución espacial en base a 772 plántulas y brinzales alrededor de 20 árboles semilleros. 


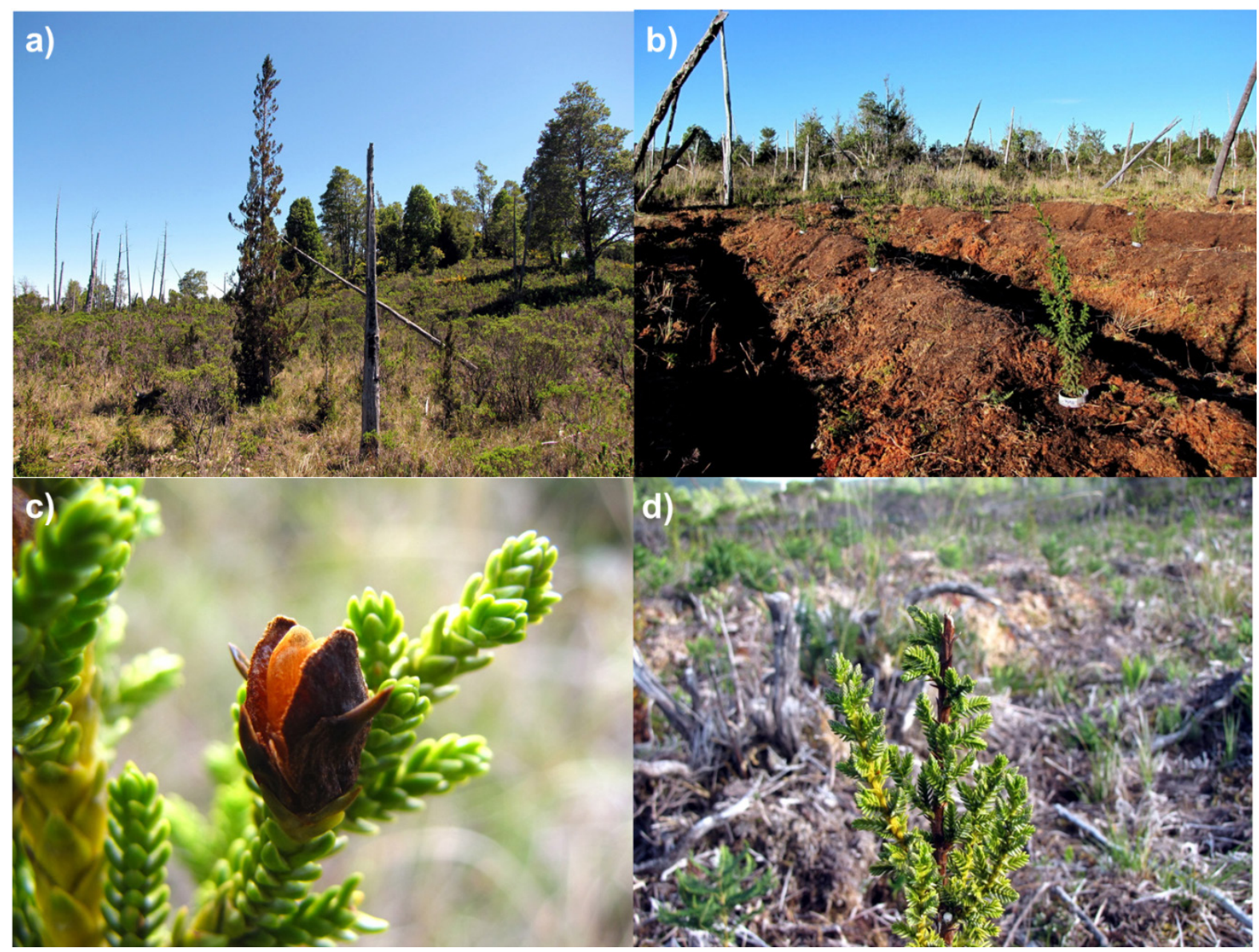

Fig. 5. Bosques turbosos afectados por incendios antrópicos en las cercanías del Lago Chaiguata, Parque Tantauco. Árboles semilleros de P. uviferum asistiendo la recuperación pasiva (a) y plantas de P. uviferum establecidas sobre camellón de

Sphagnum (b). Conos femeninos de la especie (c) y plantas dañadas por ramoneo de Pudú (d). Fotografías: Jan Bannister.

(Whisenant, 1999; Hobbs \& Harris, 2001; Hobbs \& Norton, 2004). Esto resalta la importancia de fijar objetivos de restauración y su trayectoria, de forma realista y tomando en cuenta el esfuerzo posible de realizar en la práctica (tanto económico como técnico). Esto debe definirse antes de realizar las actividades de restauración para no crear expectativas que no se puedan cumplir a futuro.

Una vez fijado el objetivo o estado deseado para una estrategia de restauración, es importante el entender los procesos que están ocurriendo en el estado del bosque actual o degradado. Con una clara visión de lo que está sucediendo en el ecosistema a restaurar, es posible el desarrollar actividades de restauración exitosas. Actualmente, los enfoques o actividades de restauración de bosques suelen dividirse en "pasivas" o la eliminación de las causantes de degradación o el uso de regeneración natural de especies arbóreas; y "activas" o el uso de regeneración artificial, quema o raleo para lograr una estructura deseada (Morrison \& Lindell, 2011; Rey Benayas et al. 2008). Es importante mencionar que muchas veces se malinterpreta el concepto de la restauración pasiva asumiendo que equivale al abandono del ecosistema. Esto es un error frecuente, ya que muchas veces para restaurar pasivamente un lugar y lograr que este se recupere se debe realizar una serie de actividades como la eliminación de ganado mediante cercos o la eliminación de malezas y especies invasoras para favorecer la regeneración natural. Para realizar esto, se debe incurrir en una serie de gastos que muchas veces no son planificados simplemente porque se considera a las acciones como pasivas. 
a)

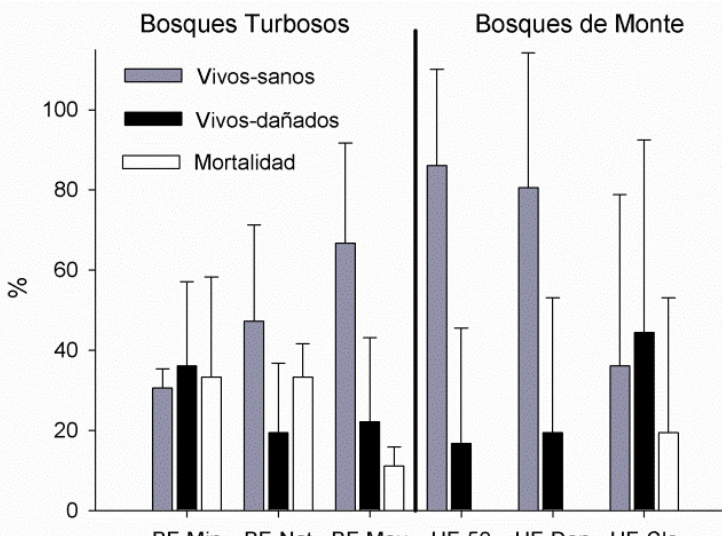

b)

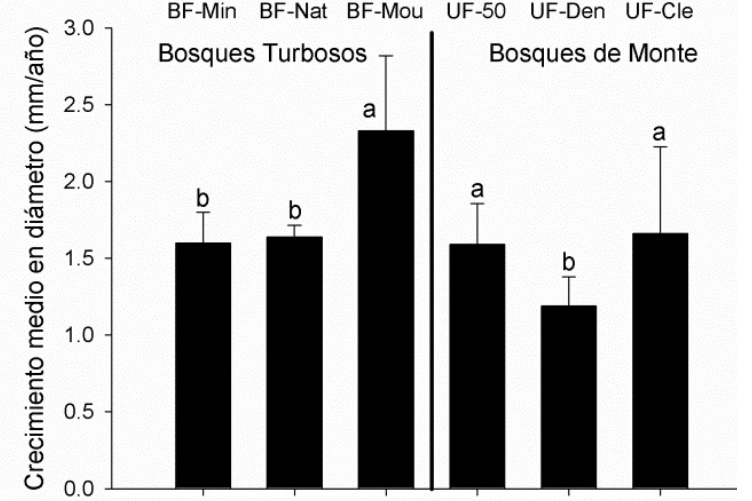

c)

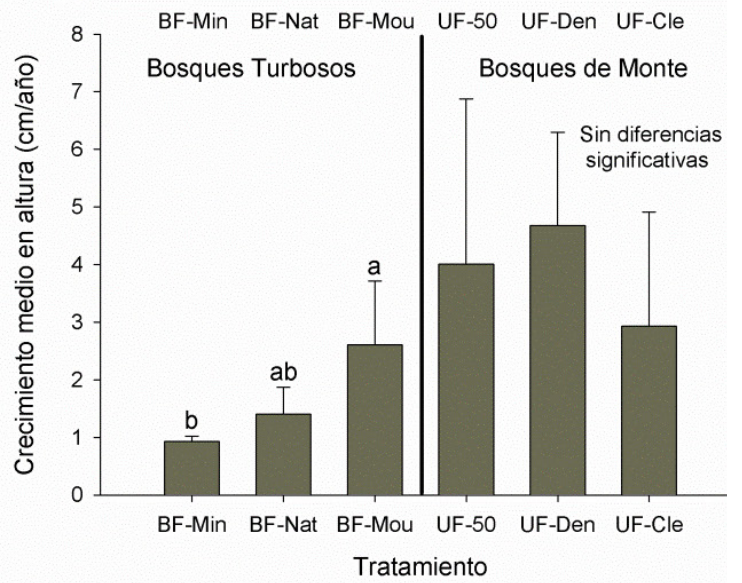

Fig. 6. Resultados de los ensayos de restauración activa con $P$. uviferum luego de 5 años en la zona del Lago Chaiguata, Parque Tantauco, Chiloé. (a) Mortalidad y daño de plantas; (b) Crecimiento medio en diámetro y (c) Crecimiento medio en altura. Letras diferentes indican diferencias significativas $(\mathrm{P} \leq 0.05$, prueba de Kruskal-

Wallis, con comparaciones post hoc basadas en prueba Mann-Whitney y el método Bonferroni). Tratamientos en bosques turbosos $(\mathrm{BF})$ : Min= suelo mineral, Nat= Natural, Mou $=$ Camellón. Tratamientos en bosques de monte (UF):

$\mathrm{Cl} e=$ talar rasa, $\mathrm{Den}=$ bajo dosel cerrado, $50=$ bajo $50 \%$ cobertura de copas.
Como ya se dijo en la sección anterior, en el caso de los bosques alterados de P. uviferum, el efecto espacial de la regeneración natural postincendio de $P$. uviferum a nivel de paisaje es extremadamente limitado (Fig. 4). Por este motivo, en los últimos años se ha explorado el potencial de la restauración activa de la especie. Para esto es de suma importancia el saber cómo plantar y cómo favorecer las plantaciones establecidas, por lo que se evaluó si la manipulación de las condiciones potencialmente limitantes puede asistir el establecimiento de las plantas. En turberas, las plantas fueron establecidas en camellones y depresiones para examinar el efecto del drenaje del suelo. En bosques de monte (con mejor drenaje), las plantas se establecieron bajo cobertura de copas y en áreas descubiertas (tala rasa), para de esta forma examinar el efecto de la luminosidad en el establecimiento. Nuestros resultados indican que luego de 5 años de desarrollo, las plantas establecidas en bosques turbosos tuvieron una menor mortalidad cuando fueron plantadas sobre camellón de Sphagnum (Figura 5b y Figura 6a) que en sustratos minerales o naturales. En el caso de bosques de monte, no se presentó mortalidad en plantas establecidas bajo cierta protección de dosel. Es importante mencionar que pese a que los indices de mortalidad fueron bajos (promedio de 0 a $40 \%$ aprox. en cada tratamiento), existieron graves daños por ramoneo de Pudú (Fig. 5d). Este daño fue mayor en sustrato mineral (bosques turbosos) y en plantas establecidas en talas rasas (bosques de monte). Esta información abre interesantes interrogantes a futuro sobre el rol del Pudú en la dinámica de estos bosques y las implicancias que tiene en ensayos de restauración de la especie. Por otro lado, los ensayos indican que plantas establecidas en bosques turbosos sobre camellones de Sphagnum presentan mayores crecimientos en diámetro y altura. En el caso de los bosques de monte, los ensayos no son concluyentes pero se nota una influencia positiva de la protección de dosel sobre el crecimiento en diámetro y altura de plantas de la especie (Fig. 6bc). De esta forma a nivel general, existe una influencia de la microtopografía (bosques turbosos) y la cobertura de copas (bosques de monte) sobre el crecimiento de plantas de P. uviferum. Estos resultados que abarcan 5 años de crecimiento, 
difieren en algunos aspectos con los presentados para el mismo ensayo por Bannister et al. (2013) con 2 años de evaluación. Esto confirma la importancia de mantener este tipo de ensayos en el largo plazo. En ese entonces, por la escasez de plantas de $P$. uviferum en viveros del sur de Chile no se pudo realizar un diseño factorial completo. Debido a esto, y considerando que actualmente el vivero de Parque Tantauco tiene sobre 12.000 plantas listas para plantación, actualmente se está evaluando en un nuevo ensayo de 6 ha el efecto protector y facilitador de matorrales secundarios de Bacharis sp. y Gleichenia sp. sobre el crecimiento y supervivencia de plantaciones de $P$. uviferum en bosques turbosos quemados.

\section{ESTRATEGIA DE RESTAURACIÓN PARA BOSQUES ALTERADOS DE PILGERODENDRON UVIFERUM}

Debido a las características de los bosques de $P$. uviferum, una estrategia de restauración para estos bosques no debiera pretender volver al ecosistema a un estado de referencia o histórico. Más bien se debería restaurar los procesos naturales para que el bosque siga su curso con la presencia de $P$. uviferum en el paisaje. Según Holl \& Aide (2011), la tasa de recuperación natural de un bosque está afectada por la resiliencia intrínseca a un disturbio, la historia de cambio de uso del suelo y las características del paisaje. En este contexto, estos autores sugieren tres preguntas que pueden ayudar a identificar objetivos para una estrategia de restauración: (1) $\mathrm{Si}$ se elige un enfoque de restauración pasiva, ¿qué resultados se pueden esperar? (2) Si una intervención es necesaria, ¿cómo y cuándo se debiera intervenir para lograr los objetivos del proyecto? y; (3) Como pueden ser usados los recursos disponibles para la restauración de la forma más eficiente a nivel de paisaje? A continuación, se aplican estas preguntas a los bosques quemados de $P$. uviferum, de tal forma de definir una estrategia de restauración para estos bosques.

Si se elige un enfoque de restauración pasiva, que resultados se pueden esperar?

El enfoque multi-escala usado en los últimos años en bosques de $P$. uviferum, en el cual se estudian los procesos ecológicos y fisiológicos esenciales que ocurren en sitios alterados $e$ inalterados de forma previa a la planificación de las actividades de restauración, ha permitido saber que si bien la regeneración desde semilleros de P. uviferum puede asistir la recuperación natural de poblaciones de la especie luego de incendios, este proceso es extremadamente limitado a escala de paisaje (Bannister et al. 2014). En este contexto una estrategia de restauración basada completamente en medidas pasivas es insuficiente, por lo que se deben buscar opciones activas para recuperar estos bosques.

Si una intervención es necesaria, ¿Cómo y cuándo se debiera intervenir para lograr los objetivos del proyecto?

Como se dijo anteriormente para restaurar bosques de $P$. uviferum es necesario intervenir el ecosistema con técnicas activas. Sin embargo, no siempre se debe optar por una u otra técnica (pasiva o activa), sino que muchas veces para ser más eficiente operativamente y tener éxito en la restauración se pueden mezclar ambos tipos de restauración. En este contexto, se puede incrementar el número de semilleros de $P$. uviferum a través de medidas concretas como el establecimiento de plantaciones en grupos (cluster planting) para complementar elementos existentes ("legados biológicos") como árboles semilleros, pequeños fragmentos y bancos de semillas dentro de una matriz de bosque quemado, y así remover el principal umbral biótico que impide la restauración pasiva. La idea con esto es tomar en cuenta los patrones de distribución de los legados biológicos y así lograr a largo plazo un efecto positivo a nivel de paisaje, por el efecto de estos "clusters" como núcleos de dispersión de semillas (Saha, 2012). Esta estrategia mixta pasiva-activa ha sido recomendada por Bannister (2012) para éstos y otros bosques degradados y quemados (e.g. bosque Norpatagónico), y actualmente se está aplicando en diferentes ensayos existentes en el Archipiélago de Chiloé.

En la figura 7 se puede observar un esquema con la estrategia de restauración mixta que está siendo aplicada en Parque Tantauco Chiloé y que 
está basada en los resultados de esta investigación. Según esta estrategia, se establecen "clusters" circulares de $6 \mathrm{~m}$ de radio con 41 plantas de $P$. uviferum homogéneamente distribuidas, y con una separación promedio entre clusters de 50 metros. Previo al establecimiento se debe realizar un diagnóstico del área a restaurar identificando espacialmente posibles legados biológicos como cipreses semilleros con regeneración, cipreses semilleros sin regeneración y cipreses machos. Luego se inicia el establecimiento de clusters cada $50 \mathrm{~m}$ y si uno de éstos queda ubicado exactamente donde existe un legado biológico, se planta alrededor de él. En el caso que el legado sea un ciprés semillero con regeneración, esta área se deja sin plantar y se destina para restauración pasiva. Esta estrategia asume que cada cluster a futuro será un núcleo de dispersión de semillas y que tendrá desde su centro una distancia de 20 $m$ de impacto positivo para la restauración pasiva (Fig. 4). Debido a esto, toda el área entre clusters se destina también a restauración pasiva. Aparte, como las plantas de $P$. uviferum al ser plantadas en sitios turbosos presentan mejores crecimientos cuando son establecidas sobre camellones, y para crearlos se requiere un injustificado alto esfuerzo, se recomienda el promover la plantación en micrositios elevados como "hummocks" y evitar el plantar en sectores de turbera anegada (Fig. 7). Por otro lado, en bosques de monte se recomienda el establecer los clusters bajo el efecto protector de matorrales o árboles de otras especies de tal forma de promover una mejor nutrición y un mayor crecimiento (Bannister et al. 2013).

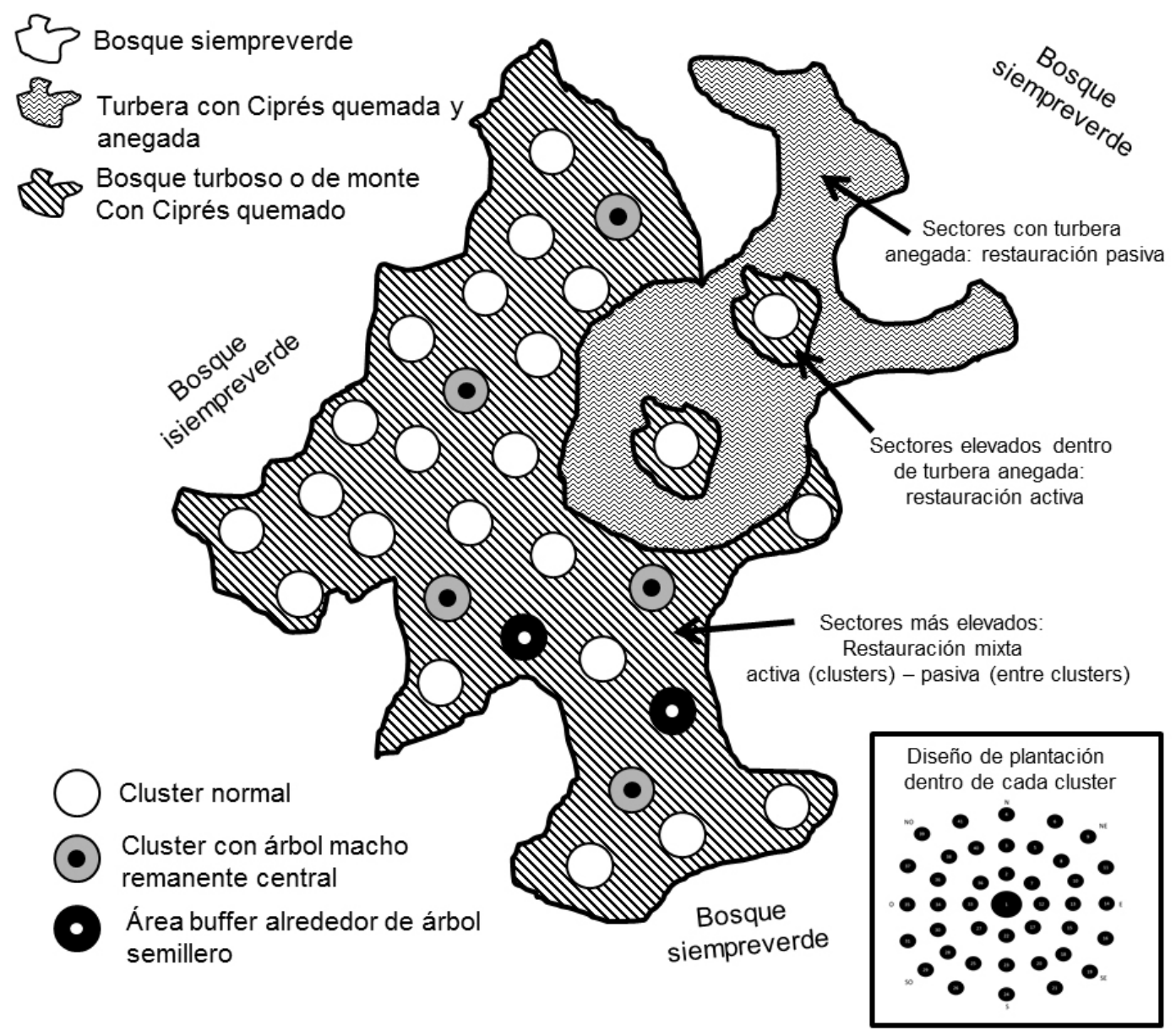

Fig. 7. Esquema que resume la estrategia de restauración mixta para bosques de Pilgerodendron uviferum desarrollada por la presente investigación y que está siendo aplicada en 20 ha a orillas del lago Chaiguata,

Parque Tantauco, Chiloé. 
Como pueden ser usados los recursos

disponibles para la restauración de la forma más eficiente a nivel de paisaje?

El problema de ecosistemas cómo los bosques de $P$. uviferum, en que la resiliencia al fuego es muy baja, presentan una alta degradación y se ubican en área remotas, es que la restauración es extremadamente costosa y los resultados son inciertos. En este contexto, la estrategia mixta de restauración sugerida, basada en regeneración natural proveniente de árboles semilleros y la plantación complementaria en grupos, sería la opción más eficiente y efectiva para restaurar los bosques turbosos y de monte alterados de $P$. uviferum en Patagonia Norte. Restaurar con un diseño de plantación en grupos puede ahorrar gran cantidad de recursos en comparación a diseños tradicionales como la plantación tradicional en hilera. Por ejemplo, en la actualidad comúnmente se ocupan en varios tipos de bosques, diseños estandarizados de 1.666 plantas/ha $(2 \mathrm{~m} \times 3 \mathrm{~m})$ o 2.500 plantas/ha $(2 \mathrm{~m} \times 2 \mathrm{~m})$. Esta investigación propone la plantación en grupos de 41 plantas cada $50 \mathrm{~m}$ de distancia, por lo que para restaurar una hectárea solo se necesitarían 164 plantas, sin considerar el área que no se planta debido a la existencia de legados biológicos. Esta estrategia de menor costo ayudaría a profesionales relacionados con el manejo de bosques, en la planificación futura de actividades de restauración con la especie. Finalmente, el enfoque multi-escala usado en esta investigación, donde previo a planificar un programa de restauración, se estudian los procesos que ocurren en bosques alterados y prístinos, puede ser adoptado para otros ecosistemas ubicados en sectores remotos, con baja resiliencia al fuego y alta degradación, y así reducir costos y mejorar la efectividad de las actividades de restauración (Bannister, 2012).

\section{LECCIONES APRENDIDAS}

El presente trabajo ha tratado de resumir los principales resultados y experiencias que se han tenido en los últimos 7 años tratando de restaurar bosques de $P$. uviferum. En este período se han tenido grandes triunfos y fracasos. Hace una década, existía la visión generalizada entre profesionales forestales de que $P$. uviferum era una especie intolerante a la sombra y que para restaurar cipresales quemados se debían crear fajas de tala rasa para remover el efecto inhibidor de los renovales secundarios de otras especies y que por otro lado se debía plantar en los sectores más anegados ya que a la especie le favorecían las condiciones extremas de humedad. Aparte, en esos años el stock de plantas de P. uviferum en Chile era tan precario que no era posible realizar operativamente un programa de restauración para la especie. Luego de 7 años, se han aclarado una serie de "mitos" sobre la ecología de la especie y ya se sabe cómo favorecer el crecimiento inicial de plantaciones con la especie en distintos sitios. Con un presupuesto limitado se ha logrado producir 12.000 plantas de P. uviferum en los viveros del Parque Tantauco las cuales están esperando a ser plantadas, y ya se iniciaron las primeras 20 hectáreas de plantación en base a la estrategia presentada en este trabajo. No todos han sido triunfos; a lo largo del tiempo se han ido encontrando en el camino una serie de problemas, entre ellos el que el restaurar estos bosques sigue siendo una actividad extremadamente costosa y de difícil financiamiento a nivel de paisaje. Además, los efectos del ramoneo de fauna nativa (e.g. Pudú), el cual es difícil de controlar y no tiene una solución obvia, aumentan la mortalidad y daño de plantas significativamente. También hay desafíos importantes, las plantaciones en cluster son una técnica silvícola que debe ser explorada en mayor profundidad a futuro tanto para los bosques de $P$. uviferum como para otros tipos de bosques. Las 20 ha de plantaciones en cluster establecidas el año 2014 aportarán con importante información ecológica y silvícola sobre la especie a una escala mayor que los experimentos anteriores (Bannister et al. 2013).

En este contexto, quizás la lección más importante que se ha aprendido en estos años, es que el recuperar bosques no es solo plantar árboles. Antes de gastar recursos en plantar o realizar alguna actividad de restauración a escala de paisaje, se debe entender los procesos ecológicos que ocurren en los bosques inalterados, se debe analizar el grado de recuperación natural de los bosques quemados, y además se debe explorar opciones de restauración a micro-escala a través 
de pequeños experimentos de bajo costo. Esto permite que al momento de ejecutar un programa macro de restauración, se pueda ser más eficiente y efectivo en el uso de los recursos y se pueda reducir la incertidumbre de los resultados. Se espera que la experiencia acumulada en estos 7 años restaurando "cipresales" y resumida en este trabajo sirva a otros investigadores y profesionales de la restauración, para que apliquen los enfoques desarrollados para esta especie en Chiloé, en otros bosques y especies de ecosistemas alterados de la Patagonia.

\section{AGRADECIMIENTOS}

Se agradece el permanente apoyo al staff de Parque Tantauco, al Instituto de Silvicultura de la Universidad de Freiburg en Alemania, a la Fundación Futuro, a la Georg Ludwig Hartig Stiftung, a una beca de doctorado DAAD-CONICYT y al proyecto PAI- CONICYT N821320007. Además se agradece a NV Carrasco-Farias, K Kremer y todos los ayudantes y estudiantes que han apoyado incansablemente esta investigación en los últimos años. Por último, este trabajo es en honor a William Charles Bannister Fabry, quien trabajó por la valoración de la construcción de ribera con madera Ciprés de las Guaitecas en el Archipiélago de Chiloé, y murió durante la preparación de este manuscrito.

\section{LITERATURA CITADA}

Aravena, J.C. (2007). Reconstructing climate variability using tree rings and glacier fluctuations in the Southern Chilean Andes. Western Ontario University, London, Ontario, Canada.

Aronson, J., Dhillion, S. \& Floc'h, E. (1995). On the need to select an ecosystem of reference, however imperfect: A reply to Pickett \& Parker. Restoration Ecology, 3, 1-3.

Bannister, J.R. (2012). Dynamics and restoration of Pilgerodendron uviferum forests on Chiloé Island, North Patagonia, Chile. Albert-Ludwigs Universität Freiburg, Freiburg, Alemania.

Bannister, J.R., Coopman, R., Donoso, P.J. \& Bauhus, J. (2013). The importance of microtopography and nurse canopy for successful restoration planting of the slowgrowing conifer Pilgerodendron uviferum. Forests, 4, 85-103.

Bannister, J.R. (2004). Estado de conservación de Pilgerodendron uviferum ( $D$ Don) Florin en el área norte de la Cordillera de Pirulil, Isla Grande de Chiloé, X región. Universidad Austral de Chile, Valdivia.

Bannister, J.R., Donoso, P.J. \& Bauhus, J. (2012). Persistence of the slow growing conifer Pilgerodendron uviferum in old-growth and fire-disturbed southern bog forests. Ecosystems, 15, 1158-1172.

Bannister, J.R., Lara, A. \& Le Quesne, C. (2008). Estructura y dinámica de bosques de Pilgerodendron uviferum afectados por incendios en la Cordillera de la Costa de la Isla Grande de Chiloé. Bosque, 29, 33-43.

Bannister, J.R., Wagner, S., Donoso, P.J. \& Bauhus, J. (2014). The importance of seed trees in the dioecious conifer Pilgerodendron uviferum for passive restoration of fire disturbed southern bog forests. Austral Ecology, 39, 204-2013.

Bullock, A. \& Acreman, M. (2003). The role of wetlands in the hydrological cycle. Hydrology and Earth System Science, 7, 358-389.

Burns, B.R. (1993). Fire-induced dynamics of Araucaria araucana-Nothofagus antarctica forest in the Southern Andes. Journal of Biogeography, 20, 669-685.

CONAF (2011). Catastro de los recursos vegetacionales nativos de Chile. Monitoreo de cambios y actualizaciones. Período $1997-$ 2011. Santiago, Chile.

Cruz, G. \& Lara, A. (1981). Tipificación, cambio de estructura y normas de manejo para Ciprés de las Guaitecas (Pilgerodendron uviferum D. Don Florin) en la isla Grande de Chiloé. Universidad de Chile, Santiago, Chile.

Donoso, C. (1981). Tipos forestales de los bosques nativos de Chile, CONAF/PNUD/FAO. ed, Investigación y Desarrollo Forestal. FAO, Chile.

Fenton, N., Lecomte, N., Legare, S. \& Bergeron, Y. (2005). Paludification in black spruce (Picea mariana) forests of eastern Canada: Potential 
factors and management implications. Forest Ecology and Management, 213, 151-159.

Franklin, J.F. (1990). Biological legacies: a critical management concept from $\mathrm{Mt}$. St. Helens. Presented at the Transactions of the Fifty-fifth North American Wildlife and Natural resources Conference, Wildlife Management Institute, Denver, CO. Washington, DC,

Franklin, J.F. \& MacMahon, J.A. (2000). Messages from a mountain. Science, 288, 11831185.

Franklin, J.F., Spies, T.A., Pelt, R.V., Carey, A.B., Thornburgh, D.A., Berg, D.R., Lindenmayer, D.B., Harmon, M.E., Keeton, W.S., Shaw, D.C., Bible, K. \& Chen, J. (2002). Disturbances and structural development of natural forest ecosystems with silvicultural implications, using Douglasfir forests as an example. Forest Ecology and Management, 155, 399-423.

Gatica, M.P.G., Rogel, P.S. \& Donaire, J.C.A. (2014). Análisis denrocronológico de árboles muertos en pie de la especie Pilgerodendron uviferum Florin (Ciprés de las Guaitecas), en el sector de Lago Toro, Provincia de Última esperanza, Region de Magallanes. Anales del Instituto de la Patagonia, 42, 5-16.

Gorham, E. (1991). Northern peatlands: Role in the carbon cycle and probable responses to climatic warming. Ecological Applications, 1, 182-195.

Gunderson, L.H. (2000). Ecological resilience - in theory and application. Annual Review of Ecology, Evolution and Systematics, 31, 425-439.

Hechenleitner, P., Gardner, M., Thomas, P., Echeverria, C., Escobar, B., Brownless, P. \& Martínez, C. (2005). Plantas amenazadas del centro-sur de Chile. Distribución, conservación y propagación. Universidad Austral de Chile y Real Jardín Botánico de Edimburgo.

Hobbs, R.J. \& Norton, D.A. (1996). Towards a conceptual framework for restoration ecology. Restoration Ecology, 4, 93-110.

Holling, C.S. (1973). Resilience and stability of ecological systems. Annual Review of
Ecology, Evolution and Systematics, 4, $1-23$.

Holling, C.S. (2001). Understanding the complexity of economic, ecological, and social systems. Ecosystems, 4, 390-405.

Holl, K.D. \& Aide, T.M. (2011). When and where to actively restore ecosystems?, Forest Ecology and Management, 261, 15581563.

Holz, A. (2010). Climatic and human influences on fire regimes and forest dynamics in temperate rainforests in southern Chile. Colorado University, USA.

Holz, A. \& Veblen, T.T. (2009). Pilgerodendron uviferum: The Southernmost tree-ring fire recorder species. Ecoscience, 16, 322-329.

Kuuluvainen, T., Aapala, K., Ahlroth, P., Kuusinen, M., Lindholm, T., Sallantaus, T., Siitonen, J. \& Tukia, H. (2002). Principles of ecological restoration of Boreal forested ecosystems: Finland as an example. Silva Fennica, 36, 409-422.

Landres, P.B., Morgan, P. \& Swanson, F.J. 1999. Overview of the use of natural variability concepts in managing ecological systems. Ecological Applications, 9, 1179-1188.

Lara, A., Amoroso, A., Bannister, J.R., Donoso, C., Gonzalez, M., Vargas, R., Smith-Ramirez, C., Arellano, G., Gutiérrez \& A.G. (2014). Sucesión y dinámica de bosques templados en Chile. En Ecología Forestal: Bases para el manejo sustentable y conservación de los bosques nativos de Chile. (pp. 323411). Ediciones UACH, Valdivia, Chile,

Lara, A., Bannister, J.R., Donoso, C., Rovere, A., Soto, D.P., Escobar, B. \& Premoli, A. (2013). Pilgerodendron uviferum (D. Don) Florin. En M. Cuneo (Ed.). Las especies arbóreas de los bosques templados de Chile y Argentina, Autoecología (pp. 8292). Valdivia, Chile,

Lara, A., Donoso, C., Escobar, B., Rovere, A., Premoli, A., Soto, D.P. \& Bannister, J.R. (2006). Pilgerodendron uviferum (D. Don) Florin. En M. Cuneo (Ed.). Las especies arbóreas de los bosques templados de Chile y Argentina, Autoecología (pp. 8291). Valdivia, Chile.

Lavoie, M., Paré, D., Fenton, N., Groot, A. 
\& Taylor, K. (2005). Paludification and management of forested peatlands in Canada: a literature review. Environmental Reviews, 13, 21-50.

Lumley, S. \& Switsur, R. (1993). Late quaternary chronology of the Taitao peninsula, southern Chile. Journal of Quaternary Science, 8, 161-165.

Martínez, O. (1981). Flora y fitosociología de un relicto de Pilgerodendron uvifera (D. Don) Florin en el fundo San Pablo de Tregua (Valdivia-Chile). Bosque, 4, 3-11.

Morrison, E.B. \& Lindell, C.A. (2011). Active or passive forest restoration? Assessing restoration alternatives with avian foraging behavior. Restoration Ecology, 19, 170-177.

Otero, L. (2006). La huella del fuego. Historia de los bosques nativos. Poblamiento y cambios en el paisaje del sur de Chile. Pehuén, Santiago, Chile.

Parish, R. \& Antos, J.A. (2006). Slow growth, long-lived trees, and minimal disturbance characterize the dynamics of an ancient, montane forest in coastal British Columbia. Canadian Journal of Forestry Research, 36, 2826-2838.

Pickett, S.T.A. \& Parker, V.T. (1994). Avoiding the old pitfalls: Opportunities in a new discipline. Restoration Ecology, 2, 75-79.

Rey Benayas, J.M.R., Bullock, J.M. \& Newton, A.C. (2008). Creating woodland islets to reconcile ecological restoration, conservation, and agricultural land use. Frontiers in Ecology and the Environment, 6, 329-336.

Roig, F. \& Boninsegna, J. (1991). Estudios sobre el crecimiento radial, basal, en altura y de las condiciones climáticas que afectan el desarrollo de Pilgerodendron uviferum. Revista Chilena de Historia Natural, 64, 53-64.

Rozzi, R., Armesto, J.J., Goffinet, B., Buck, W., Massardo, F., Silander, J., Arroyo, M.T., Russell, S., Anderson, C.B., Cavieres, L.A. \& Callicott, J.B. (2008). Changing lenses to assess biodiversity: patterns of species richness in sub-Antarctic plants and implications for global conservation. Frontiers in Ecology and the Environment, 6, 131-137.
Saha, S. (2012). Development of tree quality, productivity, and diversity in oak (Quercus robur and $Q$. petraea) stands established by cluster planting. Albert-Ludwigs Universität Freiburg, Freiburg, Alemania.

SER (2004). The SER International Primer on Ecological Restoration.

Shaw, A.J., Cox, C.J. \& Boles, S.B. (2003). Global patterns in peatmoss biodiversity. Molecular Ecology, 12, 2553-2570.

Simard, M., Lecomte, N., Bergeron, Y., Bernier, P.Y. \& Paré, D. (2007). Forest productivity decline caused by successional paludification of boreal soils. Ecological Applications, 17, 1619-1637.

Solís, C., Becerra, J., Flores, C., Robledo, J. \& Silva, M. (2004). Antibacterial and antifungal terpenes from Pilgerodendron uviferum (D. DON) Florin. Journal of the Chilean Chemical Society, 49, 157-161.

Soto, D.P., Bannister, J.R., Ríos, A. \& Le Quesne, C. (2010). Nuevos registros de poblaciones amenazadas de Pilgerodendron uviferum D.Don (Florin) en su limite norte en la Cordillera de la Costa chilena. Gayana Botanica, 67, 120-124.

Stanturf, J.A., Palik, B.J. \& Dumroese, R.K. (2014). Contemporary forest restoration: A review emphasizing function. Forest Ecology and Management, 331, 292323.

Suding, K. (2004). Alternative states and positive feedbacks in restoration ecology. Trends in Ecology and Evolution, 19, 46-53.

Szeicz, J.M., Lara, A., Díaz, S. \& Aravena, J.C. (2000). Dendrochronological studies of Pilgerodendron uviferum in southwestern South America. En Dendrocronología en América Latina (pp. 245-270). EDIUNC, Mendoza, Argentina.

Thompson, I., Mackey, B., McNulty, S. \& Mosseler, A. (2009). Forest resilience, biodiversity, and climate change a synthesis of the biodiversity/resilience/stability relationship in forest ecosystems, Technical Series. Secretariat of the Convention on Biological Diversity, Montreal.

Van Andel, J. \& Aronson, J. (2006). Restoration ecology. Blackwell Publishing. 
Villagrán, C. (1988). Late quaternary vegetation of southern Isla Grande de Chiloé, Chile. Quaternary Research, 29, 294-306.

Walker, L.R. (2011). Integration of the study of natural and anthropogenic disturbances using severity gradients. Austral Ecology, 36, 916-922.

Walter, K.S. \& Gillet, H.J. (1998). 1997 IUCN red list of threatened plants. IUCN, Gland; Switzerland and Cambridge, UK. 
